\title{
Impacto del control ciudadano en el cumplimiento normativo de los portales de transparencia estándar en empresas de saneamiento del sur del Perú
}

\author{
Impact of citizen control on regulatory compliance with standard \\ transparency portals in sanitation companies in southern Peru
}

\author{
Dennys Geovanni Calderón Paniagua \\ Universidad Nacional Mayor de San Marcos \\ Lima, Perú \\ dennyscalderon13@hotmail.com \\ https://orcid.org/0000-0002-6569-0634 \\ Grisely Rosalie Quispe Vilca \\ Universidad Nacional Mayor de San Marcos \\ Lima, Perú \\ https://orcid.org/0000-0003-0526-4366 \\ grisely_rqv@hotmail.com
}

Braulia Paniagua Quispe Ministerio de Educación del Perú

Tacna, Perú

aries123456123456@ hotmail.com https://orcid.org/0000-0003-3511-6823

Hugo Flores Choquecota

Universidad José Carlos Mariátegui

Moquegua, Perú

floreschoquecotah@gmail.com

https://orcid.org/0000-0002-2061-6131

[Presentado: 15/05/2021, Aceptado: 23/06/2021]

\section{RESUMEN}

La investigación tuvo como objetivo principal, analizar el impacto del control ciudadano en el cumplimiento normativo de los portales de transparencia estándar (PTE) de las empresas de saneamiento del sur del Perú. Respecto al tipo de investigación, se encuentra enmarcada en el tipo básica, sobre el diseño de investigación, fue de nivel descriptivo, toda vez que tiene la finalidad de generar nuevos conocimientos, al evaluar el impacto que se genera en el nivel de cumplimiento de las normas aplicables en los PTE de las empresas de saneamiento de las regiones ubicadas en el sur del Perú mediante la aplicación del control ciudadano, dentro de las normas legales de acceso a la información pública y control social, lo cual fortalece la participación ciudadana. La población y muestra estuvo constituida por las 4 empresas de saneamiento ubicadas en las regiones sur del territorio peruano, específicamente en las regiones de Arequipa, Moquegua y Tacna, asimismo, se empleó un análisis registral. Se concluye que el impacto generado luego de la aplicación del control ciudadano en la verificación del nivel de cumplimiento normativo de los PTE en las empresas de saneamiento del sur del Perú fue de un porcentaje de cumplimiento al $30.5 \%$ categorizado con la calificación de "necesitan mejorar", este resultado evidencia el bajo nivel de cumplimiento normativo y demuestra la deficiente transparencia de información pública que tienen dichas empresas hacia la población.

Palabras clave: Control, ciudadano, normatividad, transparencia, empresa.

\begin{abstract}
The main objective of the research was to analyze the impact of citizen control on the regulatory compliance of standard transparency portals (PTE) of sanitation companies in southern Peru. Regarding the type of research, this work is framed in the basic type, on the research design, it was descriptive level, since it has the purpose of generating new knowledge by evaluating the impact that is generated in the level of compliance with the norms applicable in the PTEs of the sanitation companies of the regions located in the south of Peru through the application of citizen control, within the legal norms of access to public information and social control, strengthening citizen participation. The population and sample consisted of the 4 sanitation companies located in the southern regions of the Peruvian territory, specifically in the regions of Arequipa, Moquegua, and Tacna. A registry analysis was also used. It is concluded that the impact generated after the application of citizen control in the verification of the level of regulatory compliance of the PTE in the sanitation companies of southern Peru was a compliance percentage of $30.5 \%$ categorized with the qualification of "They need to improve", this result shows the low level of regulatory compliance and demonstrates the deficient transparency of public information that these companies have towards the population.
\end{abstract}

Keywords: Control, citizen, regulations, transparency, company.

Este es un artículo de acceso abierto, distribuido bajo los términos de la Licencia Creative Commons Atribución 4.0 Internacional (CC BY 4.0). https://creativecommons.org/licenses/by/4.0/deed.es 


\section{INTRODUCCIÓN}

La transparencia de información y el derecho que tiene la ciudadanía para acceder a la información de las entidades públicas, en el marco de la modernización de la gestión pública, son instrumentos imprescindibles que permiten a la población estar informados sobre las acciones que realizan las entidades públicas y promueven la inserción de estos en la toma de decisiones, Oszlak (2012) menciona en ese contexto, la importancia que tiene la Carta Iberoamericana de Participación Ciudadana en la Gestión Pública (CLAD, 2009), que busca generar una democracia plena, que tenga como base los derechos de acceso a la información, la participación y opinión en temas públicos, concordante con la existencia de un gobierno abierto, que exige necesariamente la apertura de los gobiernos a la ciudadanía, para que ellos ejerzan libremente su derecho de acceder a la información, aunado a esto permite fortalecer la confianza en el ejercicio de la función pública que tiene la sociedad sobre las entidades, como parte de la lucha contra la corrupción en el ámbito de la transparencia de información (Rodríguez, 2003).

Aunado a esto, es visible durante los últimos años, resaltar que los recursos tecnológicos cada vez son de mayor importancia en el desarrollo de la gestión pública, empleados para fomentar la transparencia, motivar la participación de la comunidad, puesto que se convierte en la principal fuente generadora de valor público, que busca devolver la confianza del ciudadano en el adecuado y eficiente accionar del Estado, en las intervenciones que realiza para mitigar los problemas públicos que se presentan en la sociedad, con mayor fuerza durante estos últimos años, que debido a la COVID-19, y a la implementación de trabajo remoto, las entidades se han visto en la obligación de generar mecanismos de uso de medios tecnológicos rápidos y eficaces que cubran las expectativas de los ciudadanos sobre la transparencia y participación ciudadana.

El gobierno electrónico genera iniciativas importantes para que dentro del Estado se impulse medidas vinculadas a transparentar la información y fortalecer la confianza de la ciudadanía, al vincular dentro de la administración pública el uso de la tecnología, ofrecer una alternativa para que la población tenga conocimiento y monitoree la información que se encuentra almacenada en plataformas informáticas.

Al respecto, en el territorio peruano, desde el año 2017, mediante la Directiva N. ${ }^{\circ}$ 001-2017-PCM/SGP, se ha implementado el Portal de Transparencia Estándar (PTE) el cual tiene la finalidad de cumplir con el principio de publicidad de información en las entidades del Estado, y caracterizarse por ser amigable para la ciudadanía, al fortalecer la vigilancia por parte de la población en los actos que se ejecuten dentro de la administración pública, más aún cuando desde marzo de 2020, debido a los impactos que generó la pandemia de COVID-19, cambió de forma rápida la manera de vivir y trabajar, el uso de herramientas digitales fue ganando mayor importancia, tal es así que los PTE son de vital importancia puesto que permiten a los ciudadanos acceder de forma puntual y precisa a determinada información relacionada a una entidad pública, para ejercer su derecho de participación y control ciudadano (Ley 26300, 1994).

Ahora bien, aunado a los esfuerzos de lucha contra la corrupción y concordante con lo descrito en el párrafo precedente, se genera la necesidad de mencionar al control ciudadano como un aliado estratégico, que surge como una forma efectiva para que la ciudadanía participe en los asuntos del Estado, involucrándose en la gestión pública (Guillen et al, 2009), mediante la verificación y supervisión de la prestación de servicios por parte de cualquier entidad pública y en base a esta, se puedan generar cambios estructurales en la forma como se trabaja la toma de decisiones, de tal manera, que mejore los servicios brindados, el desarrollo social y genere valor público.

Sin embargo, se percibe que la ciudadanía tiene un escaso interés de vincularse con el derecho de participación mediante el control ciudadano, por falta de conocimiento y difusión de mecanismo, es por ello, que son mínimas las entidades que promueven y vinculan al ciudadano en mecanismos y acciones de participación, lo que se refleja en el descuido por algunas entidades. Al tener registrada y actualizada la información en los PTE, se evidencia el peligro que existe para la práctica de los derechos 
de participación y control ciudadano, toda vez que el ciudadano puede interpretar estos presuntos incumplimientos como parte del desarrollo común de las entidades dentro de la administración pública. Por otro lado, dentro del grupo de entidades públicas, tenemos las empresas de saneamiento, organizaciones que tienen la finalidad de brindar el servicio de agua potable, y ayudan al cierre de brechas en temas de saneamiento, sin embargo, en ocasiones, estas empresas no direccionan su accionar en brindar servicios que sean sostenibles en el tiempo, pese a que como parte del cierre de brechas en este sector, se tiene que al 2030 se logre alcanzar que el $100 \%$ de la población cuente con un servicio de calidad, así como sostenible e información que pueda ser transparentada, para que esta retome la confianza.

En este contexto, es importante conocer si las empresas públicas de saneamiento, por su relevancia dentro de la cadena de servicios públicos relacionados a la dotación de recurso hídrico a la población, alineadas al cumplimiento del sexto objetivo: agua limpia y saneamiento, de los objetivos de desarrollo sostenible al 2030 (Organización de las Naciones Unidas, 2015), vienen transparentando de forma adecuada la información citada en la directiva antes indicada, para que la ciudadanía perciba que estas brindan un servicio de calidad, toda vez que no gozan de la preferencia por parte de los usuarios sino todo lo contrario, generan disgustos, paros y protestas, como las ocurridas en la región sur en el año 2018, a causa del aumento de las tarifas (El Comercio, 2018). De lo antes mencionado, nace la oportunidad e impacto de esta investigación, ya que mediante la aplicación del control ciudadano se determinará el nivel de cumplimiento normativo de los PTE en las empresas de saneamiento del sur del territorio peruano, se evidencia el impacto que genera contar con una población capacitada, que pueda coadyuvar mediante el control ciudadano en alertar y mejorar los procesos que se desarrollan en la gestión pública.

En tal sentido, el presente trabajo consideró el siguiente objetivo: analizar el impacto del control ciudadano en la determinación del nivel de cumplimiento normativo de los PTE en las empresas de saneamiento del sur del Perú, este objetivo tiene gran relevancia puesto que permitió conocer el nivel de cumplimiento normativo de los PTE de las empresas de saneamiento en el sur del Perú, en base a la aplicación del control ciudadano, se genera una metodología descentralizada para vincular la participación ciudadana en las actividades y/o procesos que se desarrollan en la gestión pública específicamente las vinculadas con la transparencia de información, del mismo modo, permite generar el análisis sobre el adecuado desarrollo y cumplimiento normativo de las empresas de saneamiento.

Ahora bien, para esta investigación se tiene los siguientes antecedentes que evidencian la relevancia e impacto del trabajo:

Respecto al control ciudadano, Meléndez (2018), en su investigación buscó encontrar el grado de correlación existente entre la ciudadanía con el apoyo que puede brindar al control gubernamental en la región de Puno, en los procesos que se desarrollan en la gestión pública, de esta investigación se desprende que mediante los monitoreos ciudadanos que realiza la población en la zona sur de este departamento, se generó un impacto positivo, lo que se traduce en un ambiente favorable para coadyuvar a las labores de control gubernamental, por ende la lucha contra la corrupción, evidencia que la puesta en práctica del derecho de participación y control ciudadano ayuda a generar un mayor nivel de confianza por parte de la ciudadanía hacia las entidades públicas y por ende en los servicios públicos que este brinda.

De igual forma Calderón, Quispe y Pacompia (2021) desarrollaron una investigación mediante la cual por medio de la aplicación del control social que ejerce la ciudadanía como parte de sus derechos de participación ciudadana, evidenciaron el nivel de cumplimiento normativo del sistema INFObras, en la EPS Tacna S.A., esta investigación fue básica, descriptiva-explicativa, como población se consideró las obras que se encuentran registradas en el sistema INFObras, el cual es de libre acceso a la comunidad. Se concluye que posterior a la aplicación del control social, la entidad antes mencionada tiene un alto nivel de incumplimiento normativo, lo cual limita la participación de la ciudadanía de la región Tacna, es decir no les permiten conocer cómo se ejecutan las obras públicas en el marco de sus competencias. 
Respecto al cumplimiento normativo de los PTE, mediante la investigación realizada por Córdova (2020), se analizó la vinculación que existe entre las variables del PTE respecto a la gestión municipal de la provincia El Dorado, esta investigación tuvo un diseño no experimental, el cual arribó a que existe una relación positiva y lineal entre ambas variables mencionadas, con un valor de 0.881 de correlación, es decir que en la medida que se tenga la información completa y actualizada, incidirá en la gestión de la municipalidad, ya que mejora la percepción que el ciudadano tiene de esta.

Por su parte, Ruiz (2018), en su investigación desarrolló procedimientos para determinar la correlación que existe entre el gobierno abierto y el uso del PTE, en la Universidad Nacional de San Martín, para lo cual, después de aplicar una encuesta de campo a 48 servidores que laboran en la entidad antes citada, se concluye que el gobierno abierto mantiene una relación directa y significativa con el uso de los PTE, en este contexto, es importante mencionar la siguiente conclusión, se demuestra de forma fehaciente la alta correlación que existe entre ambas variables, toda vez que el gobierno abierto, uno de los tres ejes transversales de la modernización de la gestión pública, se encuentra inmerso y se vincula con la calidad de información que se transparenta a la ciudadanía.

De igual forma, Valdez et al. (2017) desarrollaron una investigación que tuvo como finalidad encontrar el grado de cumplimiento, así como de implementación de la normativa vigente respecto a la transparencia y acceso a la información pública, este trabajo fue de carácter cuantitativo y analítico, enmarcado dentro del ámbito no experimental, de igual forma lo relacionado a la muestra analizada se consideró gobierno locales del sur de Puno, como Chucuito-Juli, Yunguyo y el Collao, se concluye que las entidades materia del análisis muestran resultados caracterizados por ser insuficientes en cuanto a la información que proporcionan los PTE, esto genera que la población tenga desconfianza en las actividades que realiza la entidad en aras de transparentar la información pública y cumplir con la normativa aplicable.

En ese mismo sentido, Alarcón (2015) en su investigación buscó determinar el nivel de cumplimiento del PTE de las municipalidades de la región de Lambayeque durante el primer semestre del año 2013, para lo cual desarrolló esta investigación analítica-descriptiva, estudia la variable sin realizar ninguna alteración, la investigación tuvo como población y muestra a las 38 municipalidades tanto provinciales como distritales de la región de Lambayeque, donde se concluye que el nivel de cumplimiento es muy bajo, peor aún se determinó que algunas entidades carecen de PTE, lo tienen inactivo o con información desactualizada, lo que se traduce como una desatención hacia la comunidad, limita que la población ejerza su derecho de participación y control ciudadano e inclusive a incurrir en un incumplimiento normativo.

Sobre este aspecto, es importante mencionar la relevancia del control ciudadano que apoya en las labores de fiscalización social, que cumple un papel preponderante como fuente de información calificada, que tiene su razón de ser en el derecho de participación ciudadana enmarcado en la Constitución Política del Perú, artículo 2, que precisa, las personas tienen derecho a participar de forma individual o grupal en la vida política, social y económica de la nación. Asimismo, Mellado (2001) destaca la utilidad de la participación cotidiana de la ciudadanía en la toma de decisiones y durante la ejecución de estas, que atañen a la comunidad. De igual forma, el Banco Interamericano de Desarrollo (2004) precisa que la participación ciudadana conlleva el conjunto de procedimientos por los cuales los ciudadanos como parte activa de la sociedad, ejercer una labor de fiscalización en las actividades del Estado en los tres niveles de gobierno, se demuestra con estos autores la importancia que tiene el control ciudadano para evidenciar el buen o mal uso de los recursos públicos en la administración pública.

Finalmente, para esta investigación se ha considerado a las empresas públicas de saneamiento de la región de Tacna, Moquegua y Arequipa, por el vital servicio público que brindan a la comunidad, que debe ser de forma eficiente y transparente, ya que promueven el cierre de la brecha de saneamiento existente en el Estado Peruano. Por ello, esta investigación pretende, mediante la aplicación del control ciudadano, evidenciar el nivel de cumplimiento normativo de los PTE en estas empresas públicas, 
al considerar que para la implementación y actualización de información en esta plataforma existen marcos normativos, y su no cumplimiento pueden acarrear responsabilidades administrativas en el campo de la administración pública, sin dejar de lado la percepción sobre la calidad del servicio que tendrían los usuarios.

\section{MATERIAL Y MÉTODOS}

Según Hernández, Fernández y Baptista (2014), esta investigación se encuentra enmarcada dentro del tipo básica, presenta un nivel de investigación descriptivo, toda vez que tiene la finalidad de generar conocimiento relacionado al impacto que tiene el control ciudadano en los procesos de evaluación del nivel de cumplimiento normativo de los PTE en las empresas públicas de saneamiento en el sur del Perú. De igual forma, tiene un enfoque de investigación cuantitativa, ya que sustenta su razón de ser en la evaluación que se realiza sobre el grado de cumplimiento de los PTE en las entidades de la región sur del Perú, por último, tiene un diseño no experimental y un corte transversal, toda vez que la toma de datos fue realizada en un determinado momento.

Por otro lado, la población considerada para este trabajo se constituyó por cuatro empresas públicas de saneamiento, las cuales se encuentran ubicadas en la región Tacna, Moquegua y Arequipa, al sur del territorio peruano (Tabla 1). Asimismo, se ha considerado como muestra al $100 \%$ de estas empresas, en las cuales se aplicó el instrumento de control ciudadano, mediante el cual se obtuvo y analizó la información respecto a los PTE.

\section{Tabla 1}

Empresas públicas de saneamiento del sur del Perú

Nombre de la entidad $\quad$ Región

\begin{tabular}{lcc}
\hline $\begin{array}{l}\text { Servicio de Agua Potable y } \\
\text { Alcantarillado de Arequipa. }\end{array}$ & SEDAPAR S.A. & Arequipa \\
$\begin{array}{l}\text { Entidad Prestadora de Servicios } \\
\text { de Saneamiento Ilo S.A. }\end{array}$ & EPSILO S.A. & Moquegua \\
$\begin{array}{l}\text { Entidad Prestadora de Servicios } \\
\text { de Saneamiento Moquegua S.A }\end{array}$ & EPS Moquegua S.A. & Moquegua \\
$\begin{array}{l}\text { Entidad Prestadora de Servicios } \\
\text { de Saneamiento Tacna }\end{array}$ & EPS Tacna S.A. & Tacna \\
\hline
\end{tabular}

En relación a las técnicas empleadas en la investigación para realizar la recopilación de información, se vio por conveniente utilizar un análisis registral, al generar en primera instancia una lista de cotejo concordante con las precisiones normativas de la directiva, para seguidamente emplear la información pública disponible en los PTE, concordante con la política de participación ciudadana y transparencia de información del Estado, y se realice el control ciudadano a los PTE.

Asimismo, respecto a los materiales y herramientas empleados en la recopilación de información, se elaboró una secuencia lógica para analizar cada uno de los PTE, en base la normativa vigente que delimita la obligatoriedad de su implementación y actualización de información pública por parte de las entidades del Estado, así como la normatividad pertinente para analizar a las empresas públicas, de igual forma para la evaluación respectiva se consideró la tabla de calificación (Tabla 2) adaptada de la investigación realizada por Valdez et al. (2017), la cual permitió interpretar y categorizar los resultados obtenidos de la investigación. 
Tabla 2

Puntaje para calificar el nivel de cumplimiento

\begin{tabular}{lc}
\hline Calificación & Puntaje \\
\hline Destacado & 76 a $100 \%$ \\
Satisfactorio & 51 a $75 \%$ \\
Necesita mejorar & 26 a $50 \%$ \\
Insuficiente & 0 a $25 \%$ \\
\hline
\end{tabular}

Respecto a lo indicado líneas arriba, sobre las bases normativas empleadas para el análisis y pertinencia del trabajo de investigación, estas se detallan a continuación:

De la Ley 27785 (21 de julio de 2002), se ha considerado la citada normativa puesto que en ella se contempla la vinculación que existe entre el control social y la transparencia como parte del sistema nacional de control, enmarcado en las disposiciones finales, específicamente en la novena que precisa las definiciones básicas, a continuación, se procede a describirlas:

Control social: Es aquelderechoquetienelaciudadaníaa participar de forma activa en los procedimientos que se desarrollan dentro de la gestión y el control público, su participación es importante puesto que son considerados como información de primera fuente sobre las áreas críticas de la administración pública para identificar presuntos actos de corrupción que se pudiesen estar dando.

Transparencia: Se refiere al deber que tienen los trabajadores del Estado, de permitir que sus actuaciones relacionadas a la gestión tengan que ser informados y sustentados de forma clara a las autoridades del gobierno y a la población, con la finalidad de que estos conozcan y evalúen cómo se desarrollan los actos vinculados al buen uso de los recursos públicos, sus objetivos y metas institucionales.

De la Ley 26300 (3 de mayo de 1994), se ha considerado la citada normativa, toda vez que esta describe cuáles son los derechos de control ciudadano que ostentan todas las personas dentro del territorio peruano, la cual genera la posibilidad de aplicar este control social en las entidades públicas a nivel de los gobiernos municipales y regionales, a continuación, se precisa lo descrito:

Artículo 3.- Derechos de control ciudadano.

Se detallan los derechos de control que ostentan los ciudadanos: en el literal d), se precisa como otros mecanismos de control establecidos por la mencionada normativa, aquellos que se generen y sean aplicables a los gobiernos regionales y municipales.

De la Ley 27444 (10 de abril de 2001), se ha considerado la citada normativa, toda vez que esta describe en el principio de participación, la pertinencia de brindar información a los ciudadanos y el derecho que estos tienen de recibirla, lo descrito se precisa a continuación:

Artículo IV.- Principios del procedimiento administrativo.

1.12. Principio de participación: Respecto a este principio se detalla que las entidades deben de realizar las acciones necesarias para brindar la información necesaria y requerida por los administrados, sin necesidad de algún tipo de justificación, exceptuando aquella que afecte la intimidad personal, la que se encuentre vinculada a temas de seguridad nacional o sobre las cuales exista norma expresa que lo prohíba, asimismo, detalla que esta participación se debe realizar por un lado mediante el servicio de acceso a la información pública y por otro recepcionando la presentación de opiniones de los ciudadanos. 
Del Texto Único Ordenado de la Ley N. ${ }^{\circ} 27806$ (11 de diciembre de 2019), se ha considerado la citada normativa, toda vez que esta describe la importancia de la publicidad de la información, como fuente calificada para la ciudadanía y dentro de este contexto menciona que una forma de hacerlo es mediante los PTE, a continuación, se precisa lo descrito:

Artículo 3.- Principios de publicidad.

Hace referencia que los funcionarios públicos son responsables de facilitar el acceso a la información de las diversas áreas de las entidades, por lo que deben de custodiarla de forma ordenada, sistematizada y publicar en la medida que las leyes lo soliciten.

1. Aquella información que posea el Estado se concibe que es pública, excepcionalmente aquella contemplada por el artículo 15 de la presente normativa.

2. El Estado desarrolla acciones con la finalidad de garantizar la transparencia de información, asimismo, promoverla en todas las entidades que componen la administración pública.

3. El Estado tiene el deber de brindar la información que los ciudadanos soliciten en concordancia con el principio de publicidad.

Artículo 4.- Responsabilidades y sanciones.

El presente numeral precisa que todas las entidades del Estado, están obligadas a cumplir con lo mencionado en esta normativa, teniendo en consideración que en caso incurran en no cumplir a cabalidad con lo precisado, podrían ser sancionados por la ejecución de una falta categorizada como "falta grave".

Artículo 5.- Publicación en los portales de las dependencias públicas.

Este numeral precisa que las entidades en base a su presupuesto, de manera progresiva tienen que realizar la difusión, a través del internet, de la siguiente información:

1. Datos generales de la entidad en la cual se aprecia las disposiciones y comunicados emitidos, de igual forma, cómo está constituida su organización, el organigrama institucional, los procedimientos, su marco legal vigente, entre otros del TUO del procedimiento administrativo.

2. La información concerniente al presupuesto, que incluya los datos del presupuesto que ha sido ejecutado de los proyectos de inversión de los salarios y beneficios de funcionarios y el personal en forma general, finalmente las remuneraciones.

3. Adquisiciones de los bienes y servicios que se realicen en la entidad, la cual debe contar con el detalle de los montos comprometidos, de los proveedores, de las cantidades y las calidades de los bienes y de los servicios que fueron adquiridos.

4. De igual forma las actividades desarrolladas o que están próximas a desarrollarse con los altos funcionarios de la entidad, titulares y cargos del nivel siguiente.

5. Información adicional que la entidad considere pertinente publicar.

De la Directiva N..$^{\circ}$ 001-2017-PCM/SGP, en adelante la "directiva", se ha considerado la citada normativa toda vez que describe el marco normativo de la implementación de los PTE en las entidades del Estado Peruano, asimismo, la responsabilidad de los funcionarios en mantener actualizada la información y las responsabilidades que acarrea no realizarlo, continuación, se precisa lo descrito:

Artículo 5.- Del Portal de Transparencia Estándar PTE.

El PTE, viene a ser una herramienta informativa, que se encuentra integrada al Portal del Estado Peruano, esta contiene la gestión que desarrolla la entidad clasificada en rubros temáticos, y se presenta en una forma estandarizada para todas las entidades de la administración pública del Perú, al respecto las entidades están obligadas a publicar y mantener constantemente actualizada la información, 


\section{Calderón et al.}

concordante a los plazos que la ley de transparencia y acceso a la información pública establezca, asimismo, sin limitar que las entidades puedan publicar material adicional en los rubros establecidos.

Artículo 7.- Del Funcionario Responsable.

Las entidades deben de contar con un funcionario, quien será el responsable de la elaboración y actualización de información en el PTE, conforme a lo que establece la Ley de Transparencia y Acceso a la Información Pública.

Artículo 12.- De los contenidos de la información e iconografía amigable.

El PTE, cuenta con 10 rubros temáticos, los cuales están signados con una iconografía, es preciso indicar que si esta se encuentra de color plomo quiere decir que no se encuentra activo, evidenciándose la falta de registro o la no aplicabilidad de esta entidad, asimismo, la iconografía muestra los siguientes rubros temáticos, inicialmente se tiene los datos generales, seguidamente el planeamiento y organización, a continuación el presupuesto, posterior a este el rubro proyecto de inversión e INFObras, a continuación la participación ciudadana, seguidamente información relacionada al personal, a continuación información correspondiente de las contrataciones de bienes y servicios, seguidamente las actividades que tiene planificado el titular de la entidad o funcionarios, asimismo, lo relacionado al acceso a la información y finalmente el registro de los ciudadanos o servidores públicos, que visitan este tipo de entidades.

Artículo 13.- De la publicación obligatoria.

Este artículo precisa que, respecto a los actos administrativos, a los considerados como actos de administración interna y los documentos que deban ser publicados en el PTE, se debe consignar una nota en la que se precise la obligatoriedad de su publicación en el citado portal y los plazos en los que se debe ejecutar dicha acción.

En conclusión, las normativas antes descritas fueron consideradas como la base del análisis efectuado, respecto al impacto que se obtiene de la aplicación del control ciudadano en la verificación del cumplimiento normativo de los PTE de las empresas de saneamiento en las regiones del sur del Perú.

\section{RESULTADOS}

\section{Respecto de la implementación del PTE en las empresas públicas de saneamiento en la región sur del Perú}

Como primer aspecto a tener en cuenta dentro del análisis a los PTE de las cuatro empresas de saneamiento seleccionadas, ha sido la verificación de la implementación del PTE, concordante con el artículo 5 de la directiva, por lo que se procedió a ingresar al Portal del Estado Peruano (PEP), en el cual se verificó que efectivamente dichas empresas se encuentran correctamente registradas y presentan un PTE en estado activo, el resultado es el siguiente:

Respecto a SEDAPAR S.A., de la búsqueda y posterior revisión del PEP, se ha identificado que el nombre de la empresa figura en el PEP, por lo que se puede afirmar que esta entidad cuenta con un PTE, en tal caso, se puede verificar si registra y si tiene activado la totalidad de iconografía del sistema.

Respecto a EPSILOEDAPAR S.A., de la búsqueda y posterior revisión del PEP, se ha identificado que el nombre de la empresa figura en el PEP, por lo que, se puede afirmar que esta entidad cuenta con un PTE, a continuación, se puede verificar si registra y si tiene activado la totalidad de iconografía del sistema. 
Respecto a EPS Moquegua S.A., de la búsqueda y posterior revisión del PEP, se ha identificado que el nombre de la empresa figura en el PEP, por lo que se puede afirmar que esta entidad cuenta con un PTE, en tal caso, se puede verificar si registra y si tiene activado la totalidad de iconografía del sistema. Respecto a EPS Tacna S.A., de la búsqueda y posterior revisión del PEP, se ha identificado que el nombre de la empresa figura en el PEP, por lo que se puede afirmar que esta entidad cuenta con un PTE, en tal caso, se puede verificar si registra y si tiene activado la totalidad de iconografía del sistema.

Se concluye que al tener activado el sistema de PTE, es procedente verificar como segundo punto, los rubros, toda vez que estos deben encontrarse activos en su totalidad, de acuerdo al tipo de entidad, aspecto que será desarrollado a continuación:

\section{Respecto al análisis de la activación de los rubros temáticos del PTE}

Del total de empresas públicas de saneamiento del sur del Perú, concordante con el derecho de control ciudadano, se procedió a evaluar el cumplimiento normativo de los rubros del Portal de Transparencia Estándar en el mes de mayo 2021, en mérito al artículo 12, de la Directiva N. ${ }^{\circ}$ 001-2017-PCM/SGP alineado al contexto del gobierno abierto, como parte de los ejes transversales en el marco de la nueva gestión pública, en este sentido es preciso indicar que no todos los rubros serán aplicables al total de las entidades públicas de los tres niveles de gobierno, en el caso de las empresas de saneamiento, por ejemplo, no corresponde el rubro de participación ciudadana, al respecto se tiene lo siguiente:

Tabla 3

Verificación de la activación de la iconografía de los rubros del PTE

\begin{tabular}{clcccccccccc}
\hline $\begin{array}{c}\text { N. } \\
\text { código }\end{array}$ & Descripción & $1^{*}$ & $2^{*}$ & $3^{*}$ & $4^{*}$ & $5^{*}$ & $6^{*}$ & $7^{*}$ & $8^{*}$ & $9^{*}$ & Porcentaje \\
\hline 1 & SEDAPAR S.A. & Sí & Sí & Sí & Sí & Sí & Sí & Sí & Sí & Sí & $100 \%$ \\
2 & EPSILO S.A. & Sí & Sí & Sí & No & Sí & Sí & No & No & No & $55.56 \%$ \\
3 & EPS Moquegua S.A. & Sí & Sí & Sí & Sí & Sí & Sí & No & No & No & $66.67 \%$ \\
4 & EPS Tacna S.A. & Sí & Sí & Sí & Sí & Sí & Sí & Sí & No & No & $77.78 \%$ \\
\hline \multicolumn{7}{c}{ Porcentaje promedio }
\end{tabular}

* (1) Corresponde a los datos generales, (2) al planeamiento y organización, (3) al presupuesto, (4) información de proyectos de inversión e INFObras, (5) información relacionada al personal, (6) información relacionada a la contratación de bienes y servicios, (8) las actividades oficiales, (9) posteriormente, al acceso a la información pública, (10) y finalmente, el registro de visitas.

Referente a los resultados de la Tabla 1, en primer lugar, es preciso mencionar que para el análisis no se ha considerado el rubro de participación ciudadana puesto que no es aplicable para este tipo de empresas públicas, ahora bien, de la evaluación realizada se concluye que:

Respecto a SEDAPAR S.A., concordante con lo precisado en el numeral 12 de la directiva no presenta ninguno de los rubros temáticos de iconografía en color plomo, por lo que se denota que el $100 \%$ de estos se encuentra activo, asimismo, respecto a EPSILO S.A., presenta cuatro rubros temáticos de color plomo por lo que su cumplimiento es de $55.56 \%$, de igual forma, respecto a la EPS Moquegua S.A. presenta tres rubros temáticos de color plomo por lo que su cumplimiento es de $66.67 \%$, y finalmente respecto a EPS Tacna S.A., este presenta dos rubros temáticos de color plomo que significa inactivo, su cumplimiento es de $77.78 \%$.

Sin embargo, con los resultados obtenidos, descritos líneas arriba, no es factible realizar la calificación del nivel de cumplimiento concordante con la Tabla 2, puesto que habría que realizar un análisis sobre cada uno de los contenidos que se encuentran dentro de los rubros temáticos evaluados en la Tabla 3 , a 
continuación, se muestra los valores a los que se abordaron luego del análisis.

\section{Respecto al análisis de los contenidos por cada rubro temáticos del PTE}

Referente a los resultados de la Tabla 4, en primer lugar, hay que precisar que para el análisis se han considerado los contenidos más relevantes para la aplicación del control ciudadano descritos en la directiva, asimismo, el puntaje de calificación citados en la Tabla 2, por lo que se concluye lo siguiente:

Respecto a SEDAPAR S.A., esta presenta un porcentaje de implementación de $24 \%$, lo que significa que su nivel de cumplimiento recibe la calificación de "insuficiente".

Respecto a EPSILO S.A., esta presenta un porcentaje de implementación de $36 \%$, lo que significa que su nivel de cumplimiento recibe la calificación de "necesita mejorar".

Respecto a EPS Moquegua S.A., esta presenta un porcentaje de implementación de $48 \%$, lo que significa que su nivel de cumplimiento recibe la calificación de "necesita mejorar".

Respecto a EPS Tacna S.A., esta presenta un porcentaje de implementación de $24 \%$, lo que significa que su nivel de cumplimiento recibe la calificación de "insuficiente".

\section{Tabla 4}

Verificación de la implementación de los contenidos por cada rubro del PTE

\begin{tabular}{|c|c|c|c|c|}
\hline \multirow[b]{2}{*}{ Verificación de rubros temáticos* } & \multicolumn{4}{|c|}{ Empresa de saneamiento } \\
\hline & $\begin{array}{l}\text { SEDAPAR } \\
\text { S.A. } \\
\end{array}$ & $\begin{array}{l}\text { EPSILO } \\
\text { S.A. }\end{array}$ & $\begin{array}{c}\text { EPS } \\
\text { Moquegua }\end{array}$ & $\begin{array}{l}\text { EPS Tacna } \\
\text { S.A. } \\
\end{array}$ \\
\hline $1 \quad$ Datos generales & & & & \\
\hline $\begin{array}{l}\text { Registro datos del funcionario } \\
\text { responsable }\end{array}$ & Sí & No & Sí & No \\
\hline 1.2 Registra dirección de la entidad & Sí & Sí & Sí & No \\
\hline 1.3 Registra normas de creación de & Sí & No & Sí & No \\
\hline $1.4 \begin{array}{l}\text { Registra normas emitidas por la } \\
\text { entidad }\end{array}$ & Sí & Sí & Sí & No \\
\hline $\begin{array}{l}\text { Registra declaraciones juradas } \\
\text { de la CGR }\end{array}$ & Sí & Sí & Sí & No \\
\hline 2 Planeamiento y organización & & & & \\
\hline 2.1 Registra el ROF & No & Sí & Sí & Sí \\
\hline $2.2 \begin{array}{l}\text { Registra } \\
\text { institucional }\end{array}$ & No & Sí & Sí & Sí \\
\hline 2.3 Registra el MOF & No & Sí & Sí & Sí \\
\hline 2.4 Registra el CAP & No & Sí & Sí & Sí \\
\hline 2.5 Registra el TUPA & No & No & No & Sí \\
\hline $\begin{array}{ll}3 & \text { Presupuesto } \\
\end{array}$ & & & & \\
\hline 3.1 $\begin{array}{l}\text { Registra información } \\
\text { presupuestal }\end{array}$ & No & No & No & No \\
\hline $4 \quad$ Proyectos de inversión e INFObras & & & & \\
\hline $\begin{array}{l}\text { Registra información respecto } \\
4.1 \text { a proyectos de inversión y } \\
\text { saldos }\end{array}$ & No & No & No & No \\
\hline $\begin{array}{l}\text { 4.2 Registra información de obras } \\
\text { publicas }\end{array}$ & Sí & No & No & Sí \\
\hline
\end{tabular}




\begin{tabular}{|c|c|c|c|c|c|}
\hline 5 & Personal & & & & \\
\hline 5.1 & $\begin{array}{l}\text { Registra información de } \\
\text { montos percibidos por los } \\
\text { servidores }\end{array}$ & No & No & No & No \\
\hline 6 & Contratación de bienes y servicios & & & & \\
\hline 6.1 & $\begin{array}{l}\text { Registra el rubro de procesos } \\
\text { de selección de bienes y } \\
\text { servicios }\end{array}$ & No & Sí & Sí & No \\
\hline 6.2 & $\begin{array}{l}\text { Registra el rubro órdenes de } \\
\text { servicio }\end{array}$ & No & No & No & No \\
\hline 6.3 & Registra viáticos y pasajes & No & No & No & No \\
\hline 6.4 & Registra el rubro de publicidad & No & No & Sí & No \\
\hline 6.5 & $\begin{array}{l}\text { Registra información del rubro } \\
\text { de comité de selección }\end{array}$ & No & No & No & No \\
\hline 6.6 & $\begin{array}{l}\text { Registra información del plan } \\
\text { anual de contrataciones }\end{array}$ & No & Sí & Sí & No \\
\hline 6.7 & $\begin{array}{l}\text { Registra información de } \\
\text { penalidades aplicadas }\end{array}$ & No & No & No & No \\
\hline 7 & Actividades oficiales & & & & \\
\hline 7.1 & $\begin{array}{l}\text { Registra información del rubro } \\
\text { agenda }\end{array}$ & No & No & No & No \\
\hline 7.2 & $\begin{array}{l}\text { Registra información de } \\
\text { comunicaciones y/o notas de } \\
\text { prensa }\end{array}$ & No & No & No & No \\
\hline 8 & Acceso a la información pública & & & & \\
\hline 8.1 & $\begin{array}{l}\text { Registra la solicitud de acceso } \\
\text { a la información }\end{array}$ & No & No & No & No \\
\hline 9 & Registro de visitas & & & & \\
\hline 9.1 & $\begin{array}{l}\text { Registra información de } \\
\text { registro de visitas }\end{array}$ & No & No & No & No \\
\hline & Porcentaje de implementación & $24 \%$ & $36 \%$ & $48 \%$ & $24 \%$ \\
\hline & Porcentaje promedio & \multicolumn{4}{|c|}{$30.5 \%$} \\
\hline
\end{tabular}

* Información de mayor relevancia establecidos para el control ciudadano del anexo adjunto a la Directiva N. ${ }^{\circ} 001-2017-\mathrm{PCM} / \mathrm{SGP}$

Finalmente, luego de la aplicación del control ciudadano para evidenciar el cumplimiento normativo de los PTE de las empresas de saneamiento en el sur del Perú, se ha obtenido un porcentaje promedio de $30.5 \%$, lo que según la Tabla 2, significa una calificación de "necesita mejorar".

\section{Resumen de los resultados obtenidos de la investigación}

Para este trabajo de investigación, luego de la aplicación del instrumento de control ciudadano en los PTE correspondiente a las cuatro empresas públicas de saneamiento ubicadas en el sur del Perú (Tacna, Moquegua y Arequipa), se obtuvo un porcentaje promedio de implementación de $30.5 \%$ por lo que su calificación es de "necesita mejorar", adicionalmente en la Tabla 5, se detalla el resumen del nivel de cumplimiento por cada una de estas entidades, concordante con los resultados obtenidos en la Tabla 3 y 4 . 
Tabla 5

Resumen del nivel de cumplimiento de la implementación del PTE

\begin{tabular}{lcccc}
\hline $\begin{array}{l}\text { Empresa de } \\
\text { saneamiento }\end{array}$ & Reqión & $\begin{array}{c}\text { Activación de } \\
\text { iconografía }\end{array}$ & $\begin{array}{c}\text { Implementación de } \\
\text { contenidos }\end{array}$ & $\begin{array}{c}\text { Calificación } \\
\text { (Tabla 2) }\end{array}$ \\
\hline SEDAPAR S.A. & Arequipa & $100 \%$ & $24 \%$ & Insuficiente \\
EPSILO S.A. & Moquegua & $55.56 \%$ & $36 \%$ & Necesita mejorar \\
EPS Moquegua S.A. & Moquegua & $66.67 \%$ & $48 \%$ & Necesita mejorar \\
EPS Tacna S.A. & Tacna & $77.78 \%$ & $24 \%$ & Insuficiente \\
\hline Porcentaje & & $72.25 \%$ & $30.5 \%$ & Necesita mejorar \\
\hline
\end{tabular}

En este sentido, se observa que tanto las empresas públicas de saneamiento de la región de Tacna y Arequipa son las que presentan menor nivel de cumplimiento normativo en la implementación del PTE, toda vez que la información con la que cuentan se ha calificado como "insuficiente", en el caso de las dos empresas de saneamiento de la región Moquegua han sido calificadas como "necesita mejorar", denotando que si bien han implementado parte de la información requerida por la directiva, está aún es insuficiente, en todos los casos incumpliendo el marco normativo vigente y aplicable.

Adicionalmente, se resalta que las cuatro (100\%) empresas de saneamiento del sur del Perú, no registran información respecto a los siguientes rubros: presupuesto, personal, contrataciones (rubro de órdenes de servicios, viáticos y pasajes, comité de selección e información sobre penalidades), actividades oficiales, acceso a la información y registro de visitas.

\section{DISCUSIÓN}

El ejercicio del control ciudadano, le brinda la oportunidad a la ciudadanía de vincularse de forma activa en las acciones del Estado, al estar atentos a los procesos y cuestiones relacionados a la sociedad, de igual forma brinda al ciudadano acceso público de información del Estado y sus políticas, para monitorear si sus acciones se desarrollan con el fin supremo de generar valor público, a través de las operaciones gubernamentales que ejecuta, concordante con Márquez y Távara (2010), quienes precisan que la vinculación de la ciudadanía en temas públicos constituye un derecho fundamental, cuya práctica debe ir afianzándose a medida que avanzan los años.

Al respecto, el objetivo fue analizar el impacto del control ciudadano en la determinación del nivel de cumplimiento normativo de los PTE en las empresas de saneamiento del sur del Perú, la cual fue contrastada mediante la aplicación del instrumento de control en los PTE, del cual, se obtuvo que la implementación de los OTE se encuentran en un $30.5 \%$, con la calificación de "necesita mejorar", ello quiere decir que el deficiente cumplimiento normativo de los PTE de las empresas de saneamiento limita que la población pueda tener acceso a la información, que por norma debe ser pública, lo que genera un desmedro en percepción, en el valor público que proyectan estas entidades hacia la ciudadanía. Asimismo, no cumplen con los objetivos de la directiva, por consiguiente, limitan el fortalecimiento del control ciudadano y la transparencia de información pública.

Cabe mencionar, que al identificar que las empresas de saneamiento no cumplen con la implementación y actualización de información acorde a la directiva, esta situación acarrearía en concordancia con el artículo 4 de la directiva, posibles sanciones por parte de los funcionarios y servidores responsables, los cuales tendrían que ser evaluados por la entidad, para que dentro del marco de sus competencias tome las acciones adecuadas conforme a ley, por otro lado, se está frenando la utilización de los PTE para generar confianza a la ciudadanía, distinto a otros lugares en el mundo, donde los sistemas tecnológicos, potencia la divulgación de información y acercamiento del ciudadano e inclusión en la toma de decisiones. 


\section{CONCLUSIONES}

Primeramente, de la verificación a la implementación de los PTE por las empresas de saneamiento en el sur del Perú, se concluye que estas se encuentran debidamente registradas y en estado operativo para aplicar el control ciudadano.

El impacto generado por la aplicación del control ciudadano en la verificación del nivel de cumplimiento normativo de los PTE en las empresas públicas del rubro saneamiento del sur del Perú dio como resultado, que estas empresas obtengan un porcentaje de cumplimiento de $30.5 \%$ categorizado con la calificación de "necesitan mejorar", este resultado evidencia el bajo nivel de cumplimiento normativo y demuestra la deficiente transparencia de información que tienen dichas empresas hacia la población.

La investigación permitió conocer adicionalmente, que las empresas SEDAPAR S.A. de Arequipa y EPS Tacna S.A. son las que menor porcentaje de implementación presentan, solo con el $24 \%$ cada una, calificado como "insuficiente", en segundo lugar, la EPSILO S.A. con un porcentaje de implementación de $36 \%$ y en primer lugar la EPS Moquegua S.A. con un porcentaje de implementación de $48 \%$, estas dos últimas con una calificación de "necesita mejorar". Asimismo, se evidencia que ninguna de estas empresas de saneamiento ha logrado obtener una calificación de "satisfactorio" o "destacado", por lo que se evidencia la poca predisposición para transparentar la información pública concordante a lo precisado en la directiva.

De igual manera, la aplicación del control ciudadano permitió evidenciar que dentro de la implementación de los PTE de las empresas de saneamiento del sur del Perú, existen rubros críticos, en los cuales el $100 \%$ de las empresas públicas de saneamiento consideradas como parte de la muestra analizada, no registraron ningún tipo de información, los rubros son los siguientes: presupuesto, personal, contrataciones (rubro de órdenes de servicios, viáticos y pasajes, comité de selección e información sobre penalidades), registro de visitas, acceso a la información y actividades oficiales.

Finalmente, la presente investigación permitió generar un instrumento constituido por la Tabla 2, que permite calificar el nivel de cumplimiento normativo de los PTE. Asimismo, la Tabla 3, que permite caracterizar qué rubros del PTE son aplicables a las empresas públicas de saneamiento, la Tabla 4 que permite evidenciar si los rubros del PTE se encuentran activos o inactivos, y la Tabla 5 que permite realizar una análisis a profundidad del contenido que se encuentra dentro de cada uno de los rubros del PTE, para que pueda ser replicado por la ciudadana y sociedad civil organizada, concordante a las políticas de participación en los procesos de la gestión pública, con el fin de ejercer su derecho de control ciudadano, alertar a las entidades competentes los incumplimientos detectados, y coadyuvar a mejorar la gestión pública de este tipo de entidades.

\section{REFERENCIAS}

Banco Interamericano de Desarrollo (2004). Estrategias para promover la participación ciudadana en las actividades del Banco. https://dhls.hegoa.ehu.eus/documents/4608

Calderón D, Quispe, G, y Pacompia, J. (2021). Control social en el cumplimiento normativo del sistema INFOBRAS en la Entidad Prestadora de Servicios de Saneamiento Tacna S.A. Economía \&Amp; Negocios, 3(1), 29-40. https://doi.org/10.33326/27086062.2021.1.1039

CLAD. (2009). Carta Iberoamericana de Participación Ciudadana en la Gestión Pública.

Contraloría General de la República (21 de julio de 2002). Ley 27785, Ley Orgánica del Sistema Nacional de Control y de la Contraloría General de la República.

Córdova, Z. (2020). Portal de transparencia estándar y la gestión municipal en la provincia de El Dorado, región San Martín, 2020. (Tesis de Maestría). Universidad Cesar Vallejo, Perú; recuperado de: https://hdl.handle.net/20.500.12692/50510 
Decreto Supremo N. ${ }^{\circ}$ 021-2019-JUS (11 de diciembre de 2019). Texto único ordenado de la Ley $N .^{o}$ 27806, Ley de transparencia y acceso a la información pública.

El Comercio (2018). EPS ¿son rentables las empresas prestadoras de servicio de saneamiento? Disponible en: https://elcomercio.pe/economia/peru/eps-son-rentables-empresas-prestadorasservicios-saneamiento-noticia-615230-noticia/

Guillen, A; Sáenz, K; Badii, M; y Castillo, J. (2009). Origen, espacio y niveles de participación ciudadana. Daena: International Journal of Good Conscience

Hernández, R., Fernández, C. y Baptista, M. (2014). Metodología de la investigación. McGraw-Hill Educación.

Ley 26300 (3 de mayo de 1994) Ley de los derechos de participación y control ciudadano.

Márquez, J. y Távara, G. (2010). Participación ciudadana y buen gobierno. Mesa de concertación para la lucha contra la pobreza. Primera edición, marzo del 2012, Lima - Perú. p.4.

Meléndez, A. (2018). Nivel de aplicación de la norma sobre veeduría ciudadana para el control gubernamental en los gobiernos locales provinciales de la 123 zona sur de la región Puno2016 [Tesis de maestría, Universidad Nacional del Altiplano, Puno, Perú].

Mellado, R. (2001), Participación ciudadana institucionalizada y gobernabilidad en la Ciudad de México. México: Plaza y Valdés. http://www.scielo.org.mx/scielo.php?script=sci_ nlinks\&ref=777941\& $\mathrm{pid}=$ S1870-0063200900010000400027\&lng=es

Organización de las Naciones Unidas (2015). Proyecto de documento final de la cumbre de las Naciones Unidas para la aprobación de la agenda para el desarrollo después de 2015, 12 de agosto de 2015. Recuperado de: http://www.un.org/es/comun/docs/?symbol=A/69/L.85

Oszlak, O. (2012, septiembre). “Gobierno Abierto. Promesas y desafios.” Voces en el Fénix”. Año 3. Revista N 18: La Sombra del Poder. Estado II. Plan Fénix. Buenos Aires.

Resolución Ministerial N.o 035-2017-PCM (2017). Directiva N. a 001-2017-PCM/SGP, Lineamientos para la implementación del Portal de Transparencia Estándar en las entidades de la administración pública

Rodríguez, M. (2003, julio - diciembre). El acceso a la información pública en Argentina. Derecho Comparado de la Información $\mathrm{N}^{\circ}$ 2. Pp. 111 - 143. Méjico

Ruiz, J. (2018). Gestión del gobierno abierto y su relación con el uso del portal de transparencia estándar en la Universidad Nacional de San Martín, 2018. (Tesis de Maestría). Universidad Cesar Vallejo, Perú; recuperado de: https://hdl.handle.net/20.500.12692/31636

Valdez, K., Melo, M., Carpio, J., Sotomayor, J. y Yépez, K. (2017). Evaluación de la implementación de los portales de transparencia estándar en concordancia con la normativa en las municipalidades provinciales del sur de Puno. Revista de Investigaciones de la Escuela de Posgrado de la UNA PUNO, 6(4), 362-369. https://doi.org/10.26788/epg.v6i4.141 